\title{
Fifty Years of Nuclear Astrophysics: A Foreword
}

\author{
John Lattanzio ${ }^{\mathrm{A}}$ \\ A Centre for Stellar and Planetary Astrophysics, Monash University, Melbourne VIC 3800, Australia. \\ Email: john.lattanzio@sci.monash.edu.au
}

Abstract: In 2007 the field of nuclear astrophysics celebrated its 50th birthday.

Fifty years have passed since the publication of the seminal works of Burbidge, Burbidge, Fowler, and Hoyle ( $\left.\mathrm{B}^{2} \mathrm{FH}\right)$, and by A. G. W. Cameron. These ground-breaking papers combined astronomy, astrophysics and nuclear physics in a way not done before. As a result, a new field was born, demanding attention from those in the three parent disciplines. Since then, nuclear astrophysics has led our quantitative understanding of the origin of matter. This multi-disciplinary effort encompasses input from all relevant fields, as experimental nuclear physicists discuss reaction rates with observational astronomers who turn their telescopes to stars to try to constrain the experiments. Throughout, the theorists try to make sense of the data, from laboratories and telescopes, while using improving computational resources to tackle problems of ever-increasing complexity.

Much of the original work for $\mathrm{B}^{2} \mathrm{FH}$ was performed at Caltech's Kellogg Radiation Laboratory, ${ }^{*}$ and it was here that over 120 of the world's premier nuclear astrophysicists met ${ }^{\dagger}$ in July 2007 to celebrate the anniversary, from its historical beginnings, through the present and with an eye to the future. No formal publication resulted from that meeting but a (biased) selection of authors were asked to prepare a review based on their papers presented at the meeting. We present these six papers in this issue (Burbidge 2008; Dillmann et al. 2008; Haxton 2008; Qian 2008; Salpeter 2008; Zinner 2008). I am particularly proud of the opportunity to publish the historical papers by Geoffrey Burbidge and Ed Salpeter.

I wish to record my thanks to those authors who took the time in their busy schedules to prepare these papers.

\section{References}

Burbidge, G. 2008, PASA, 25, 30. doi:10.1071/AS07029

Dillmann, I., Domingo Pardo, C., Käppler, F., Mengoni, A., \& Sonnabend, K. 2008, PASA, 25, 18. doi:10.1071/AS07043

Haxton, W. C. 2008, PASA, 25, 44. doi:10.1071/AS07041

Qian, Y.-Z. 2008, PASA, 25, 36. doi:10.1071/AS07042

Salpeter, E. E. 2008, PASA, 25, 1. doi:10.1071/AS07036

Zinner, E. E. 2008, PASA, 25, 7. doi:10.1071/AS07039 